\title{
Refractory Hyperlactatemia and Hypoglycemia in an Adult with Non- Hodgkin's Lymphoma: A Case Report and Review of the Warburg Effect
}

\author{
Zainab Al Maqrashi ${ }^{a} \quad$ Mary Sedarous $^{a} \quad$ Avinash Pandey $^{b} \quad$ Catherine Ross $^{c}$ \\ Ahraaz Wynea \\ aDepartment of General Internal Medicine, McMaster University, Hamilton, ON, Canada; \\ bMcMaster University Medical School, Candidate 2021, McMaster University, Hamilton, \\ ON, Canada; 'Department of Pathology and Molecular Medicine, McMaster University, \\ Hamilton, ON, Canada
}

\section{Keywords}

Hyperlactatemia · Warburg effect · Metabolic acidosis · Oncological complications · NonHodgkin's lymphoma

\begin{abstract}
Lactate is a byproduct of anaerobic glycolysis, and hyperlactatemia is commonly seen in critically ill patients. We report a case of an elderly male presenting with undifferentiated constitutional symptoms, anemia, thrombocytopenia, severe lactic acidosis, refractory hypoglycemia, and a newly detected abdominal mass. A dedicated workup ruled out infectious etiologies and revealed metastatic non-Hodgkin's lymphoma. This study explores etiologies of type B lactic acidosis in oncology patients, with a focus on Warburg's effect, and its potential for prognostication.
\end{abstract}

\section{Introduction}

Lactate is a degradation product of glucose in anaerobic conditions. Under optimal conditions with adequate oxygen supply, glycolytic production of pyruvate is followed by its conversion into acetyl CoA which ultimately undergoes mitochondrial oxidation in the Krebs cycle (tricarboxylic acid cycle) to produce adenosine triphosphate. During anaerobic conditions, mitochondrial oxidation cannot occur, and pyruvate is preferentially diverted through 
the Cori cycle (lactic acid cycle) in an attempt to produce cellular energy. Eventually, the resultant lactate is cyclically converted to glucose by the liver [1].

There are 2 major subtypes of hyperlactatemia causing lactic acidosis: type A and type B. The distinction is based on the underlying pathophysiology. Type A lactic acidosis is observed in cases of oxygen debt resulting from the supply-demand mismatch. Examples include shock, regional tissue hypoperfusion (mesenteric ischemia, compartment syndrome, and burns), cellular hypoxia (carbon monoxide poisoning and methemoglobinemia), and increased metabolic demand (grand-mal seizures, vigorous exercise, and severe shivering/hypothermia). Type B lactic acidosis is linked to various pathologies affecting critical metabolic pathways such as decreased gluconeogenesis (liver disease), accelerated glycolysis (malignancyinduced adrenergic states), impaired Krebs cycle (genetic diseases, drug-mediated mitochondrial toxicity, and deficiency of cofactors), and iatrogenic exogenous loads [2, 3].

Lactic acidosis is one of the most commonly reported acid-base abnormalities in critically ill patients [4]. In a retrospective cohort study of intensive critical unit admissions, mortality among patients with metabolic acidosis was significantly higher than those with no metabolic acidosis ( 45 vs. $25 \%, p<0.001$ ). Moreover, lactate was associated with higher fatal outcomes (56\%) compared to other metabolic acidosis subtypes (hyperchloremic acidosis: 29\%; other anions: $39 \%$ ) [5]. Given that lactic acidosis is predominantly driven by systemic or organlimited hypoperfusion in critically ill patients, resuscitative efforts are generally focused on optimization of oxygen delivery through volume expansion, inotropic support, and broadspectrum antibiotics. In this study, we present a case of a rare cause of persistent type B lactic acidosis in a patient with concomitant hypoglycemia without neuroglycopenic symptoms, secondary to a physiological phenomenon called Warburg's effect.

\section{Case Report}

A 65-year-old man with a past medical history of mild chronic obstructive pulmonary disease presented to the emergency department with a 9-month history of weight loss, lack of appetite, and generalized fatigue in the absence of localizing visceral symptoms. On examination, he appeared to be dehydrated and cachexic with fluid-responsive hemodynamics. The patient's vitals on arrival to the emergency department were heart rate 109 beats per minute, blood pressure $78 / 54 \mathrm{~mm} \mathrm{Hg}$, respiratory rate 17 per min, and oxygen saturation $97 \%$ on room air. After receiving $3 \mathrm{~L}$ of crystalloids, his blood pressure rose to $93 / 69 \mathrm{~mm} \mathrm{Hg}$. On inspection, there were no stigmata of chronic liver disease with the exception of numerous ecchymoses on his extremities. Occasional coarse crepitations were auscultated bilaterally in his lower lung zones. He had nontender splenomegaly and a palpable nonpulsatile firm periumbilical mass. No lymphadenopathy, scrotal, or pedal swelling was noted.

Initial workup revealed anemia (62 g/L), thrombocytopenia $\left(48 \times 10^{9} / \mathrm{L}\right)$, hypoglycemia $(2.9 \mathrm{mmol} / \mathrm{L})$, and severely elevated anion gap metabolic acidosis $\left(\mathrm{pH} 7.22, \mathrm{pCO}_{2} 26, \mathrm{HCO}_{3}\right.$ 10 , and anion gap 26) felt to be secondary to hyperlactatemia (18.8 mmol/L). Table 1 documents key laboratory results during his hospitalization. He was admitted to a high-dependency unit where he received aggressive fluid-resuscitation with crystalloids, blood transfusion, and broad-spectrum antibiotics. Workup for infection was negative including cultures of blood and urine. D-lactate level was normal. Chest and abdominal CT showed diffuse cervical-mediastinal lymphadenopathy, nodular consolidative changes in the left upper lobe, and a $3.9 \mathrm{~cm} \times 1.8 \mathrm{~cm}$ mesenteric mass, suspicious for lymphoproliferative disorder versus lymphomatous spread of another suspected primary malignancy (Fig. 1).

The patient continued to have persistent hypoglycemia (2.5-3.9 mmol/L) without neuroglycopenic symptoms. Workup for hypoglycemia (Table 2) was also grossly unyielding for

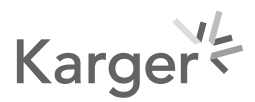


Table 1. Summary of key laboratory findings during hospitalization

\begin{tabular}{llllllllll}
\hline Variable & Reference range & Pre-admission & D0 & D3 & D6 & D12 & D15 & D17 & D19 \\
\hline Hemoglobin, g/L & $130-180$ & 99 & 62 & 78 & 72 & 77 & 72 & 62 & 90 \\
Platelets, $\times 10^{9} / \mathrm{L}$ & $150-400$ & 81 & 48 & 35 & 43 & 36 & 40 & 24 & 12 \\
Absolute neutrophils, $\times 10^{9} / \mathrm{L}$ & $2.0-7.5$ & 2.0 & 3.5 & 1.7 & 2.0 & 1.8 & 2.3 & 5.1 & 4.6 \\
$\mathrm{INR}$ & $0.8-1.1$ & 1.1 & 1.0 & - & - & - & 1.6 & 1.6 & 1.3 \\
$\mathrm{APTT}, \mathrm{s}$ & $22-35$ & 24 & 36 & - & - & - & - & 120 & 51 \\
$\mathrm{pH}$ & $7.35-7.45$ & 7.39 & 7.22 & 7.19 & 7.26 & 7.28 & 7.31 & $<6.8$ & 6.91 \\
$\mathrm{HCO}_{3}, \mathrm{mmol} / \mathrm{L}$ & $22-26$ & 23 & 11 & 9 & 13 & 12 & 16 & 6 & 9 \\
Anion gap & $<12$ & 9 & 26 & 25 & 23 & 20 & 24 & 28 & 27 \\
Glucose, mmol/L & $3.5-11$ & 5.8 & 2.9 & 2.8 & 2.9 & 3.6 & 3.1 & 2.6 & 1.7 \\
Lactate, mmol/L & $0.7-2.1$ & 3.5 & 18.8 & 16.8 & 14.9 & 17.1 & 19.9 & 26.7 & 36.0 \\
Urate, mmol/L & $218-459$ & - & 454 & 397 & - & - & 612 & 597 & 161 \\
LDH, U/L & $120-250$ & - & 179 & - & 236 & - & 246 & 265 & 756 \\
Creatinine, $\mu \mathrm{mol} / \mathrm{L}$ & $60-110$ & 62 & 82 & 71 & 58 & 53 & 88 & 160 & 208 \\
Urea, mmol/L & $3.6-9.2$ & 5.2 & 9.8 & 5.8 & 6.1 & 7.8 & 21.9 & 27.6 & 23.1 \\
Potassium, mmol/L & $3.5-5.2$ & 4.4 & 4.5 & 3.6 & 3.7 & 4.2 & 5.0 & 5.3 & 6.6 \\
Calcium, mmol/L & $2.10-2.60$ & 2.17 & 2.08 & 1.93 & - & - & 2.33 & 2.25 & 1.87 \\
Phosphate, mmol/L & $0.90-1.52$ & 1.01 & 1.09 & 0.59 & 0.52 & - & 1.52 & 3.69 & 2.91 \\
\hline
\end{tabular}

hepatic dysfunction, endogenous insulin, or major hormonal deficiency (with the exception of mild hypothyroidism [TSH $7.11 \mathrm{mIU} / \mathrm{L}$ plus fT4 $7.3 \mathrm{pmol} / \mathrm{L}]$; not felt likely to be the cause). He required continuous dextrose infusion to achieve normoglycemia, despite oral food intake.

The patient's mesenteric mass was not easily accessible without high risk of complications given its proximity to the vasculature, and so he underwent bronchoscopy and gastrointestinal endoscopies to obtain tissue samples. These did not yield any malignant cells. Bronchoalveolar lavage also did not yield any organisms. A bone marrow aspiration and biopsy on day 11 of admission, delayed due to initial patient apprehension, eventually diagnosed CD20positive non-Hodgkin's lymphoma: diffuse large B-cell lymphoma with CD10 and cyclin D1 overexpression (Fig. 2).

His lactate levels climbed steadily (Fig. 3), and he continued to require dextrose infusions to maintain normoglycemia despite initiation of prednisone $100 \mathrm{mg}$ orally daily. He acutely deteriorated between days 18 and 19 when he developed encephalopathy, nosocomial pneumonia from aspiration, sepsis, tumor lysis syndrome, and eventual multiorgan failure. After transfer to the intensive care unit, mechanical intubation, initiation of vasopressors, and continuous renal replacement therapy, his family eventually opted to withdraw care on day 19 , and he passed away shortly thereafter.

\section{Discussion}

\section{Introduction}

Hyperlactatemia is defined as a serum lactate level exceeding $2.0 \mathrm{mmol} / \mathrm{L}$ without definite acidemia. Lactic acidosis is hyperlactatemia with acidemia $\mathrm{pH}<7.35$ [2]. This continuum results from an imbalance between production and clearance of lactate. In normal physiologic conditions, lactate is the byproduct of anaerobic respiration. Lactate

\section{Karger's}




\section{Case Reports in Oncology}

\begin{tabular}{l|l}
\hline Case Rep Oncol 2021;14:1159-1167 \\
\hline DOI: 10.1159/000517658 & $\begin{array}{l}\text { ○ 2021 The Author(s). Published by S. Karger AG, Basel } \\
\text { www.karger.com/cro }\end{array}$ \\
\hline
\end{tabular}

Al Maqrashi et al.: Refractory Hyperlactatemia and Hypoglycemia in a Patient with Lymphoma

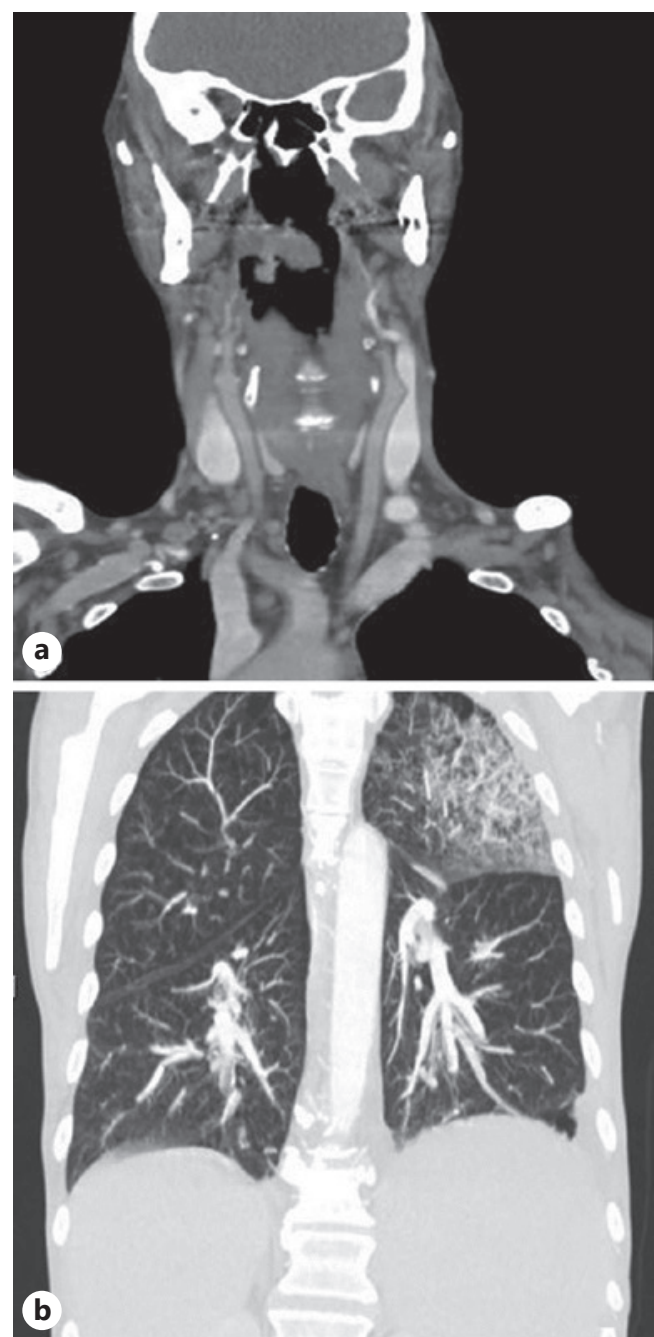

Fig. 1. Contrast CT of the head, neck, thorax, abdomen, and pelvis. Imaging showed cervical and mediastinal lymphadenopathy (A), extensive centrilobular and paraseptal emphysema with patchy bilateral nodular consolidative opacities in both lungs (B), and a new bulky confluent central mesenteric soft tissue and splenomegaly (C).

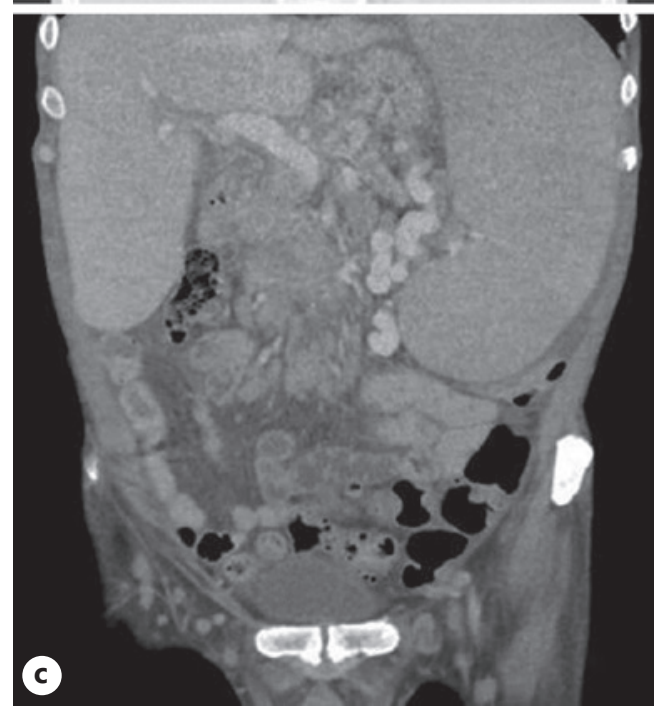

can also be produced from the deamination of alanine as well. On the clearance side, lactate is largely recycled by the liver to glucose followed by renal clearance of $25-30 \%$ of the circulating load $[1,6]$. 


\section{Case Reports in Oncology}

Table 2. Hypoglycemia workup

\begin{tabular}{l|l}
\hline Case Rep Oncol 2021;14:1159-1167 \\
\hline DOI: 10.1159/000517658 & $\begin{array}{l}\text { ○ 2021 The Author(s). Published by S. Karger AG, Basel } \\
\text { www.karger.com/cro }\end{array}$ \\
\hline
\end{tabular}

Al Maqrashi et al.: Refractory Hyperlactatemia and Hypoglycemia in a Patient with Lymphoma

\begin{tabular}{lll}
\hline Test & Reference range & Result \\
\hline Markers of liver function & & \\
$\quad$ ALT, U/L & $0-49$ & 21 \\
AST, U/L & $18-54$ & 29 \\
Bilirubin, umol/L & $<21$ & 6 \\
$\quad$ Albumin, g/L & $42-50$ & 31 \\
$\quad$ INR & $0.8-1.1$ & 1.1 \\
Ketones & & Negative \\
Insulin, pmol/L & $<120$ & $<6$ \\
C-peptide, pmol/L & $298-2,350$ & 253 \\
AM cortisol, nmol/L & $200-660$ & 472 \\
Thyroid function & & \\
TSH, mIU/L & $0.47-4.68$ & 7.11 \\
Free thyroxine, pmol/L & $10.8-28.2$ & 7.3 \\
\hline
\end{tabular}
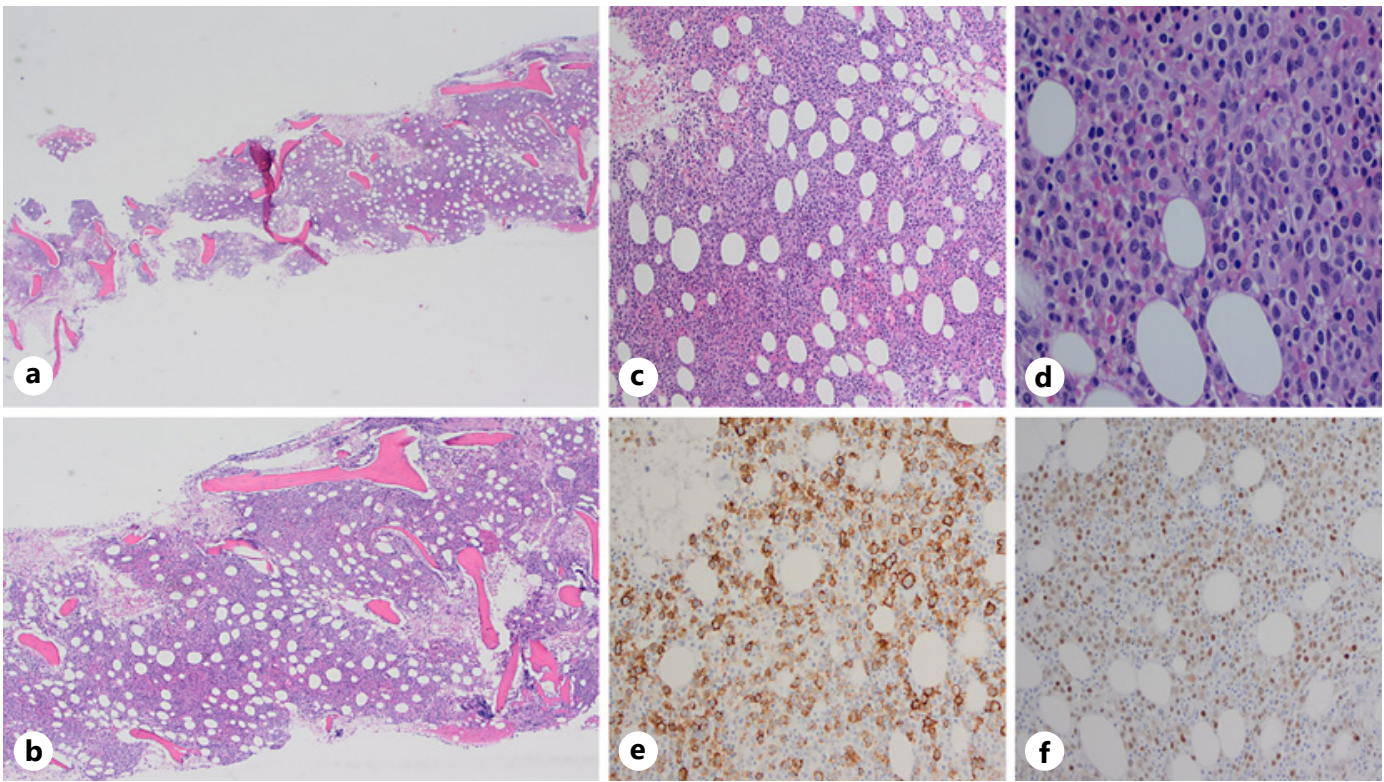

Fig. 2. Histopathology of bone marrow aspirate and biopsy. Bone marrow at $\times 20(\mathbf{A})$ and $\times 100$ (B) showing increased cellularity with an increase in atypical mononuclear cells. Bone marrow at $\times 200$ (C) and $\times 400$ (D) showing marked increase in atypical large lymphocytes. E CD20 (×200) identifies the numerous B cells. F Cyclin D1 (×200) overexpression is noted.

\section{Pathophysiology and Mechanisms of Disease}

Excess lactate is observed in 2 situations, secondary to direct tissue hypoperfusion (type A), leading to overproduction, and in its absence (type B). While cancer patients are particularly vulnerable to sepsis and shock-related illnesses, type B hyperlactatemia can also be seen due to a peculiar phenomenon called Warburg's effect. Warburg described this phenomenon in 1956 when malignant ascitic cells were compared with healthy tissue. A unique shift in a tumor's metabolic physiology to "aerobic glycolysis" regardless of the local oxygenation status was noted $[7,8]$. In advanced cases, as glucose is sequestered and preferentially driven 


\section{Case Reports in Oncology}

Case Rep Oncol 2021;14:1159-1167

Al Maqrashi et al.: Refractory Hyperlactatemia and Hypoglycemia in a Patient with Lymphoma

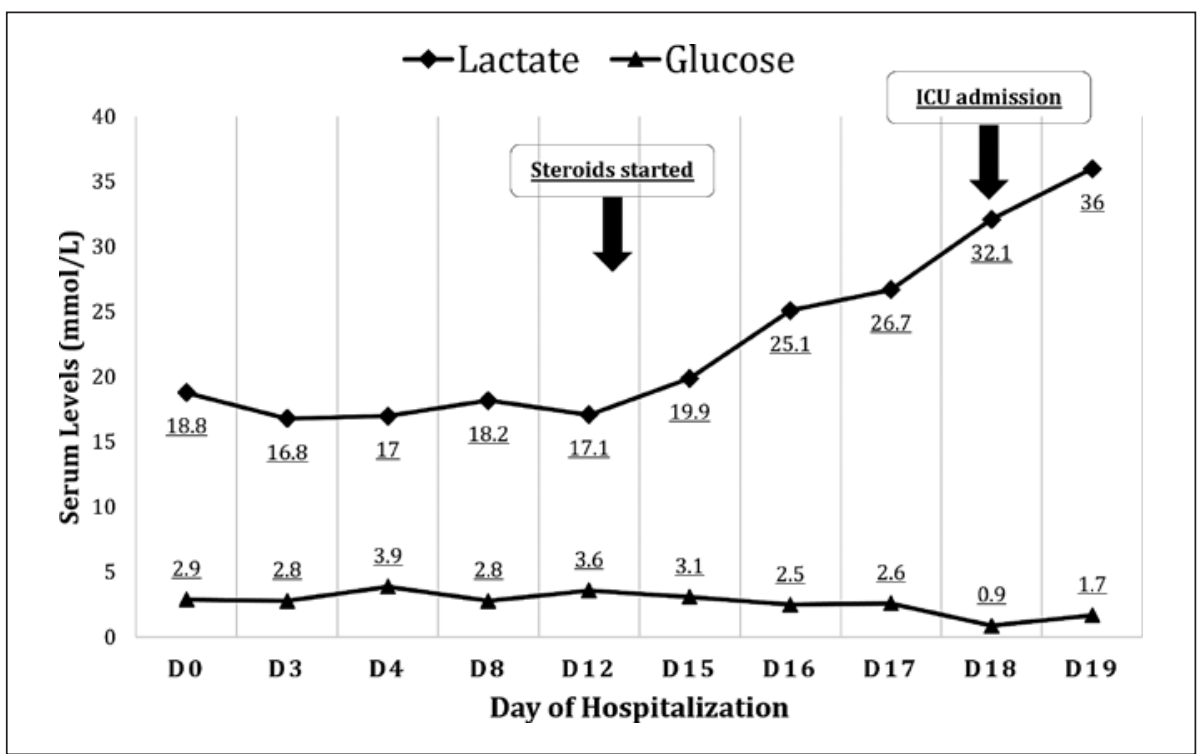

Fig. 3. Trend of serum lactate and glucose (mmol/L) in relation to days of hospitalization.

through the pyruvate-lactate pathway, overwhelming glucose consumption occurs with eventual hypoglycemia and lactate production. Moreover, bulky malignancies with high proliferative rates that exhaust the local oxygenation capacity via angiogenesis will create relative hypoxia and subsequent lactatemia. Other purported mechanisms include decreased hepatic and/or renal clearance in cases of advanced hepatic malignancy, extensive malignant deposits, or cytotoxic therapy. Additionally, thiamine and riboflavin are known enzymatic cofactors of aerobic carbohydrate metabolism, and their deficiency may result in lactate accumulation $[9,10]$. Both conditions are seen in cancer patients and linked to chronic malnutrition (decreased intake) and hypercatabolism (overutilization) [11, 12].

We now understand that Warburg's effect plays an important role in sustaining carcinogenesis and cellular proliferation for tumors [13]. Clinical implications of lactic acidosis stem from electrochemical alterations leading to cerebral injury, muscular dysfunction including respiratory fatigue, predisposition to cardiac arrhythmia, and circulatory collapse [14]. Similarly, hypoglycemia causes complications due to sympathoadrenal activation, neurocognitive dysfunction, and the generation of a pro-inflammatory response [15].

\section{Description of Trends from the Literature}

It is not uncommon for aggressive hematologic and highly undifferentiated solid organ malignancies to present with lactic acidosis that is unresponsive to resuscitative efforts [16, 17]. In fact, a few case reports have reported worsening of lactic acidosis when dextrose infusions are started for the treatment of concurrent and asymptomatic hypoinsulinemic hypoglycemia [18]. These cases support Warburg's effect and preferential shuffling of glucose through glycolytic metabolic pathways. Proposed mechanisms by which this can occur include upregulated expression of membrane-bound glucose uptake transporters, hexokinase, pyruvate kinase (M2-PK), insulin-like growth factors, and inflammatory cytokines (namely, TNF- $\alpha$ ) which attenuate enzymatic activity leading to lactate overproduction [19-21]. These protein mediators have been a heavy focus for cancer research, as therapeutic targets and prognostic tools. Biomarkers of predicting resistance to traditional therapies (chemotherapy, radiation, and immunology) have been successfully demonstrated in gastrointestinal and breast malignancies $[22,23]$. In a model studying key proteins implicated in the metabolism 
of testicular germ cell tumors, overexpression of certain molecular markers (CAIX: carbonic anhydrase IX; HKII: hexokinase II) was associated with statistically significant rates of recurrence and high-risk features (nonseminoma tumors, advanced stage) [24]. Furthermore, even more interestingly so, ketogenic diets have been recognized for their tumor-suppressive benefits. The fat-rich, carbohydrate-restricted composition of such diets directs metabolism toward ketolysis which leads to a reduction in circulating serum glucose levels. This in turn has an anti-proliferative effect through depriving neoplastic cells of their major metabolic substrate (i.e., glucose) as well as through negative feedback of anabolic proteins such as insulin and insulin-like growth factors [25].

Ruan et al. [26] demonstrated that in established lymphoma patients presenting with elevated lactate levels, 35\% (18/51) had proven progression of lymphoproliferative disease histologically or radiologically. All patients had aggressive non-Hodgkin lymphoma. Among $78 \%(14 / 18)$ of the type B lactic acidosis group, the estimated median time to death was 6 days. They concluded that after exclusion of sepsis and major ischemia, lactate level that fails to normalize in $48 \mathrm{~h}$ of initiated resuscitative measures and/or elevated lactate dehydrogenase $\geq 2 \times$ upper limit of normal are potentially indirect biochemical signs of poor overall survival and disease progress or relapse. While lactic acidosis by itself is a significant predictor of morbidity and mortality in hematological malignancies, there is a paucity of evidence describing prognostic associations with Warburg's effect $[9,14]$.

Management of lactic acidosis and hypoglycemia in suspected or confirmed cases of malignancy should account for causative processes (sepsis, drug toxicity, and liver failure), which in these patients are not always mutually exclusive. Despite that, supportive measures are eventually futile without timely administration of directed cytoreductive therapy, even in patients with good performance status and functional capacity. A nonsystemic review of case reports of lactic acidosis in lymphoma patients concluded that the only successful intervention in patients that induced biochemical resolution and cancer remission was not, unsurprisingly, chemotherapy. The earliest documented lactate normalization was achieved by 15 $\mathrm{h}$ after induction [27]. Bicarbonate infusions have also been utilized in conjunction with renal replacement therapy for severe cases until a diagnosis is made and definitive management can be started. Both modalities work to normalize extracellular $\mathrm{pH}$ and reverse the toxic effects of acidemia on cardiac function. Besides, the safety of bicarbonate infusions has been scrutinized due to eventual paradoxical increases in lactate production while failing to correct intracellular acidity $[28,29]$.

Our patient's lactic acidosis and hypoglycemia persisted despite resuscitative fluids, antibiotics, and dextrose infusions and worsened following administration of steroids. This can likely be attributed to multiple factors. First, the development of tumor lysis syndrome which has been associated with steroid initiation in high disease burden states in malignancies [30, 31]. Second, the gradual onset of sepsis from aspiration and pneumonia almost certainly contributed. Finally, with the onset of multiorgan failure, hepatic dysfunction would have ensued and worsened the lactic acidosis and hypoglycemia eventually resulting in circulatory collapse and his demise.

\section{Conclusion}

Warburg effect is a paraneoplastic phenomenon of hyperlactatemia and hypoglycemia that should be considered on the differential for acutely ill patients with suspected malignancy, especially those with a high disease burden. Patients demonstrating Warburg's effect likely have a higher risk of morbidity, mortality, and potential conversion to tumor lysis syndrome. Management should be targeted toward the underlying malignancy along with

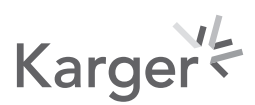


supportive fluids, dextrose, and close monitoring for infectious complications. Warburg's effect may present a unique window of opportunity for salvage therapy; however, more research is needed to understand its prognostic significance.

\section{Statement of Ethics}

Written informed consent was obtained from the next of kin for publication of this case report and any accompanying images. Based on our local policies, institutional approval was not required for case report(s) that included 3 or less patients where no systematic investigation was conducted. However, the case was written within standards of reporting, and no identifiers were included.

\section{Conflict of Interest Statement}

The authors have no conflicts of interest to declare.

\section{Funding Sources}

The authors have not declared a specific grant for this research from any funding agency in the public, commercial, or not-for-profit sectors.

\section{Author Contributions}

Z.A. and S.M. worked on procurement of data, drafting the original manuscript, and formulation of the index. A.P. was involved in proofreading and manuscript design. A.W. secured informed consent and critically reviewed the original manuscript. C.R. provided relevant graphs and images.

\section{Data Availability Statement}

All data generated or analyzed during this case report are included in this article and its supplementary material files. Further enquiries can be directed to the corresponding author.

\section{References}

1 Madias NE. Lactic acidosis. Kidney Int. 1986;29(3):752-74.

2 Fall PJ, Szerlip HM. Lactic acidosis: from sour milk to septic shock. J Intensive Care Med. 2005 Sep;20(5):25571.

3 Zosel A, Egelhoff E, Heard K. Severe lactic acidosis after an iatrogenic propylene glycol overdose. Pharmacotherapy. 2010;30(2):219.

4 Jung B, Rimmele T, Le Goff C, Chanques G, Corne P, Jonquet O, et al. Severe metabolic or mixed acidemia on intensive care unit admission: incidence, prognosis and administration of buffer therapy. a prospective, multiple-center study. Crit Care. 2011;15(5):R238.

5 Gunnerson K, Saul M, He S, Kellum J. Lactate versus non-lactate metabolic acidosis: a retrospective outcome evaluation of critically ill patients. Crit Care. 2006;10(1):R22.

6 van Hall G. Lactate kinetics in human tissues at rest and during exercise. Acta Physiol. 2010;199(4):499-508.

7 Warburg 0. On the origin of cancer cells. Science. 1979;123(3191):309-14.

\section{Karger'א}




\section{Case Reports in Oncology}

\begin{tabular}{l|l}
\hline Case Rep Oncol 2021;14:1159-1167 \\
\hline DOI: 10.1159/000517658 & $\begin{array}{l}\text { @ 2021 The Author(s). Published by S. Karger AG, Basel } \\
\text { www.karger.com/cro }\end{array}$ \\
\hline
\end{tabular}

Al Maqrashi et al.: Refractory Hyperlactatemia and Hypoglycemia in a Patient with Lymphoma

8 Vander Heiden M, Cantley L, Thompson C. Understanding the Warburg effect: the metabolic requirements of cell proliferation. Science. 1979;324(5930):1029-33.

9 Friedenberg AS, Brandoff DE, Schiffman FJ. Type B lactic acidosis as a severe metabolic complication in lymphoma and leukemia: a case series from a single institution and literature review. Medicine. 2007;86(4): 225-32.

10 Masood U, Sharma A, Nijjar S, Sitaraman K. B-cell lymphoma, thiamine deficiency, and lactic acidosis. Proc (Bayl Univ Med Cent). 2017 Jan;30(1):69-70.

11 Van Zaanen HC, van der Lelie J. Thiamine deficiency in hematologic malignant tumors. Cancer. 1992;69(7): 1710-3.

12 Thakur K, Tomar SK, Singh AK, Mandal S, Arora S. Riboflavin and health: a review of recent human research. Crit Rev Food Sci Nutr. 2017;57(17):3650-60.

13 Liberti M, Locasale J. The Warburg effect: how does it benefit cancer cells? Trends Biochem Sci. 2016;41(3): 211-8.

14 Ruiz JP, Singh A, Hart P. Type B lactic acidosis secondary to malignancy: case report, review of published cases, insights into pathogenesis, and prospects for therapy. Scientific World Journal. 2011;11:1316-24.

15 Kalra S, Mukherjee JJ, Venkataraman S, Bantwal G, Shaikh S, Saboo B, et al. Hypoglycemia: the neglected complication. Ind J Endocrinol Metab. 2013;17(5):819.

16 Sillos E, Shenep J, Burghen G, Pui CH, Behm FG, Sandlund JT. Lactic acidosis: a metabolic complication of hematologic malignancies. Cancer. 2001;92(9):2237-46.

17 Gharia B, Seegobin K, Mahida H, Shaikh M, Matthews Hew T, Pham D. Fatal type B lactic acidosis associated with metastatic colorectal cancer: a case report with review of literature, pathogenesis, and treatment. J Investig Med High Impact Case Rep. 2018 Jan;6:2324709618788101.

18 Dogan E, Erkoc R, Sayarlioglu H, Alici S, Dilek I, Alici O. Fatal lactic acidosis due to leukemic transformation in a patient with non-Hodgkin's lymphoma: case report. Adv Ther. 2005;22(5):443-6.

19 Dürig J, Fiedler W, de Wit M, Steffen M, Hossfeld DK. Lactic acidosis and hypoglycemia in a patient with highgrade non-Hodgkin's lymphoma and elevated circulating TNF- $\alpha$. Ann Hematol. 1996;72(2):97-9.

20 Mathupala SP, Ko YH, Pedersen PL. Hexokinase II: cancer's double-edged sword acting as both facilitator and gatekeeper of malignancy when bound to mitochondria. Oncogene. 2006;25(34):4777-86.

21 Christofk HR, Vander Heiden MG, Harris MH, Ramanathan A, Gerszten RE, Wei R, et al. The M2 splice isoform of pyruvate kinase is important for cancer metabolism and tumour growth. Nature. 2008;452(7184):230-3.

22 Zhou M, Zhao Y, Ding Y, Liu H, Liu Z, Fodstad O, et al. Warburg effect in chemosensitivity: Targeting lactate dehydrogenase-A re-sensitizes taxol-resistant cancer cells to taxol. Mol Cancer. 2010;9(1):33.

23 Sawahama H, Ishimoto T, Sugihara H, Miyanari N, Miyamoto Y, Baba Y, et al. Clinical impact of the Warburg effect in gastrointestinal cancer (Review). Int J Oncol. 2014;45(4):1345-54.

24 Bonatelli M, Silva ECA, Cárcano FM, Zaia MG, Lopes LF, Scapulatempo-Neto C, et al. The Warburg effect is associated with tumor aggressiveness in testicular germ cell tumors. Front Endocrinol. 2019;10:417.

25 Tran Q, Lee H, Kim C, Kong G, Gong N, Kwon SH, et al. Revisiting the Warburg Effect: diet-based strategies for cancer prevention. BioMed Res Int. 2020;2020:1-9.

26 Ruan GJ, Gandhi S, Abeykoon JP, Schram S, Habermann TM, Sandefur BJ, et al. Elevated serum lactate in patients with lymphoma: it is not always infection. Mayo Clin Proc Innov Qual Outcomes. 2021;5(2):423-30.

27 Chan FH, Carl D, Lyckholm LJ. Severe lactic acidosis in a patient with b-cell lymphoma: a case report and review of the literature. Case Rep Med. 2009;2009:1-6.

28 Fraley DS, Adler S, Bruns FJ, Zett B. Stimulation of lactate production by administration of bicarbonate in a patient with a solid neoplasm and lactic acidosis. N Engl J Med. 1980;303(19):1100-2.

29 Forsythe SM, Schmidt GA. Sodium bicarbonate for the treatment of lactic acidosis. Chest. 2000;117(1):260-7.

30 Brault C, Zerbib Y, Delette C, Marc J, Gruson B, Marolleau JP, et al. The Warburg effect as a type B lactic acidosis in a patient with acute myeloid leukemia: a diagnostic challenge for clinicians. Front Oncol. 2018;8:232.

31 Griffin D, Myadam R, Patel P. Steroid-induced lactic acidosis in diffuse large B-cell lymphoma. Cureus. 2020; 12(3):e7446. 